\title{
ANALYSIS AND EVALUATION OF TECHNOLOGICAL AND OPERATIONAL ALTERNATIVES FOR HEAVY OIL GATHERING SYSTEMS IN THE FIELDS OF CASTILLA AND CHICHIMENE - COLOMBIA
}

\author{
Mario Castillo \\ Department of Industrial Engineering \\ Universidad de los Andes \\ Bogotá, Colombia \\ E-mail: mcastill@uniandes.edu.co \\ José-Antonio Padilla \\ ECOPETROL S.A. \\ Bogotá, Colombia \\ E-mail: jose.padilla@ECOPETROL.com.co \\ Fredy Niño \\ ECOPETROL S.A. \\ Bogotá, Colombia \\ E-mail: Fredy.Nino@ECOPETROL.com.co \\ Andrés Zárate \\ ECOPETROL S.A. \\ Bogotá, Colombia \\ E-mail: andres.zarate@ECOPETROL.com.co \\ Jorge Bustamante \\ ECOPETROL S.A. \\ Bogotá, Colombia \\ E-mail: Jorge.Bustamante@ecopetrol.com.co, \\ Luis-Jose Novoa \\ Department of Industrial Engineering \\ Universidad de los Andes \\ Bogotá, Colombia \\ E-mail: lu-novoa@uniandes.edu.co \\ Juan-Carlos Gutiérrez \\ Department of Industrial Engineering \\ Universidad de los Andes \\ Bogotá, Colombia \\ E-mail: juan-gu1@uniandes.edu.co
}

\begin{abstract}
The exploitation of heavy oil has become an important business opportunity for all oil companies worldwide. The leading Colombian oil company, ECOPETROL, has several projects related to the production of extra heavy oils. This paper presents the main activities conducted and the most significant results obtained in an interdisciplinary project developed by ECOPETROL and the Universidad de Los
\end{abstract}


Andes. The main objective of the project was to develop, in advance, a general methodology and support models for the analysis and evaluation of transportation alternatives for heavy crude in two particular fields, before all the relevant information from these fields was available. ECOPETROL assessed and provided accurate information that lead to a final transportation alternative recommendation. In order to include all the technical aspects of the problem, such as chemical and physical characteristics of the fields, a multidisciplinary work team was organized to develop the different types of models needed for the decision analysis. Three models were developed, a hydraulic model (multiphase model), a financial model, and a qualitative model (Analytic Hierarchy Process), that interact to achieve the main objective. In a first step, these models were applied to a generic unit of analysis, where simulated wells are distributed in a generic manner, and then the results were successfully used in the real fields. The paper begins with a problem description, followed by the depiction of models development and simulation design. Finally, it presents a summary of the main results and conclusions.

Keywords: analytic hierarchy process, heavy oil gathering systems, heavy oil multiphase flow

\section{Introduction}

The exploitation of heavy crude oil has become an important business opportunity for all oil companies worldwide. The leading Colombian oil company, ECOPETROL, has several projects related to the production of extra heavy crude. The configuration of the gathering system and the transportation mechanism for collecting the fluids are two of the most difficult problems for companies, where heavy oil fields have not been developed and little information on wells and fluids is available.

This paper presents the main activities conducted and the most significant results obtained in an interdisciplinary project developed by ECOPETROL and the Universidad de Los Andes. This oil company needed to analyze and evaluate different alternatives for well collection systems in two particular fields, Castilla and Chichimene, which shall have, in a near future, more than three hundred production wells. The main objective of the project was to develop a general methodology and support models for the analysis and evaluation of transportation alternatives for heavy crude in the Castilla and Chichimene fields. ECOPETROL assessed and provided accurate information that lead to a final transportation alternative recommendation.

Due to the particular characteristics of the fields considered (temperature, pressure and water content, among others), that differentiate them from many other areas, the selection of a transportation mechanism for the crude is not an easy task. The topography of the area must be also taken into account in order to select the proper pipe configuration. Due to the important amount of capital invested in operational and infrastructure costs, and the uncertainty of some crucial variables, the project became a complex and urgent matter for the company.

In order to include all the technical aspects of the problem, such as chemical and physical characteristics of the fields, a multidisciplinary work team was organized in order to provide the relevant information for the problem, and to develop the different types of models needed for the decision analysis. Before deciding the type of model to be used in the analysis, it is necessary to accurately describe the problem. This means, to include its major components, the relevant variables, the actors or financial agents involved in the decision process, and the available alternatives, among others. Castillo (Castillo, 2008) designed the methodology below. Figure 1 summarizes the main steps of the general methodology that was used in the structuring and analysis of the problem.

The article begins with the problem description, followed by the depiction of models development and simulation design, and concludes with a summary of the main results and conclusions. 


\section{Problem description}

ECOPETROL must collect and measure fluids from the wells of the Castilla and Chichimene fields. These fluids must then be transported from the wellhead to a central fluid treatment station, called T2. This process must be financially and technically efficient. The most appropriate oil gathering alternative, from an operational, technological and financial point of view, must be implemented.

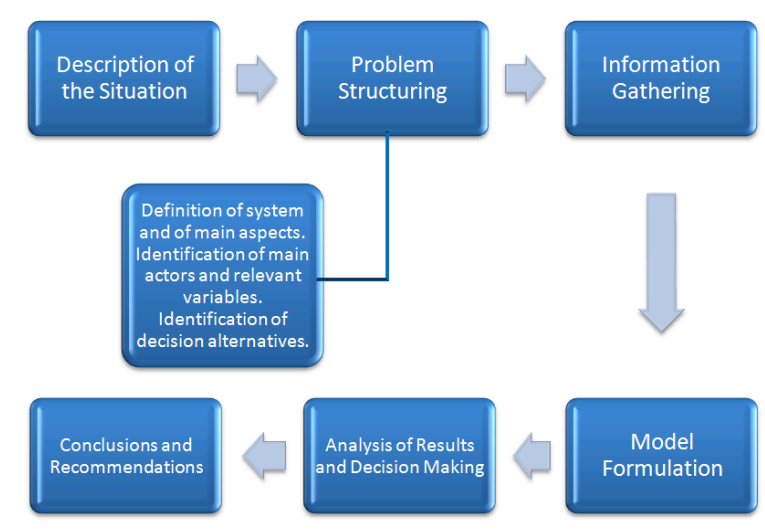

Figure1. General Methodology.

The following design considerations were taken into account:

- The wells of the Castilla and Chichimene fields that presently are producer wells of the Guadalupe K1 - K2 formation, will be producer wells of the San Fernando formation.

- The deposit development polygons for the Castilla and Chichimene fields require up to 171 production wells in Chichimene and 136 in Castilla. These wells shall be evenly distributed in the areas demarcated by deposits.

- The schedule for starting the well production is known in advance.

\section{Problem structuring}

\subsection{Main aspects of the problem}

The following aspects were taken into account for the problem analysis, model development and evaluation of alternatives:

Technical: refers to the technical specifications for the design and implementation of the oil collection alternatives in the Castilla and Chichimene fields. Variables associated with the well conditions and fluid transportation to the treatment station were considered.

Financial: refers to the investment of capital, operation and maintenance costs, and measures of financial performance of the different alternatives analyzed.

Social: refers to the probable impact of land acquisition or other project activities on neighbor communities. This aspect includes all activities that may affect the welfare of these communities. 
Environment: refers to potential environmental problems that could occur as a result of the implementation of the different alternatives. This aspect includes possible accidents caused by spillage, outbreak of fire, and pollution or emissions, among others.

\subsection{Relevant variables}

The main variables identified were:

- Distance from the clusters to the treatment station.

- Variables associated with field conditions such as wellhead pressures, oil, water and gas flow rates; API, temperature, volume and viscosity, among others.

- Variables associated with fluid transportation, such as pipe diameter and length.

- Energy consumption.

- Availability of naphtha, gas and / or water.

- Investments.

- Operation and maintenance costs.

\subsection{Restrictions and decision criteria}

The initial decision criteria to evaluate the different alternatives for fluid gathering, identified by the decision making group, were the following:

- Financial Performance: refers to the financial performance of different alternatives, as measured by the expected amount and risk of total cost.

- Theft: refers to collateral aspects that may affect the corporate image and increase in some way the vulnerability of the field's operation. Thus, the security aspect should be considered in a technical and financial context as part of planning ahead.

- Feasibility of Construction: refers to the level of complexity of the infrastructure to be installed associated with each alternative.

- Operational Complexity: refers to the degree of difficulty of the system's operation associated with each alternative. Such complexity may be related to monitoring and operating of the equipment required.

- Maintainability and Integrity: Refers to maintaining the equipment in operating conditions.

- Ensuring the Source: refers to guaranteeing the provision of supplies and raw materials required for the operation of the collection system under each alternative (energy, gas and naphtha, for example).

- Technological Development (Technological Domain): refers to ECOPETROL's level of knowledge and expertise in operating the different alternatives.

- Complexity of land acquisition: level of complexity of the negotiation with owners of adjoining land that should be acquired for the implementation of each alternative.

- Time of execution and delivery of equipment: refers to the time of implementation for a particular alternative and the time required for the delivery of equipment needed for its operation.

\subsection{Structuring of decision alternatives}

For purposes of defining the alternatives, two decision variables were considered: the mechanism used to transport fluids from the clusters to the treatment station and the type of settlement of the gathering system or pipe configuration (arrangements). Table 1 presents the initial alternatives considered by the decision making group.

\subsection{Unit of analysis}

The unit of analysis for the evaluation of alternatives was defined as a quadrant of the field with a given number of wells. A quadrant is a set of wells arranged in several clusters in order to perform the different 
analysis. These wells must be distributed in a generic manner, so that the quadrant is as representative as possible of the real situation of the fields. In the analysis of the quadrant, information resulting from the exploratory wells was taken into account. Figure 2 represents a possible generic quadrant for analysis and the arrangements that were considered in the alternatives.

Table 1. Decision alternatives.

\begin{tabular}{|c|c|c|}
\hline Mechanism & Alternative & Arrangement description \\
\hline \multirow{3}{*}{ Power } & A1 & $\begin{array}{l}\text { Individual and independent lines from cluster location to treatment } \\
\text { station. }\end{array}$ \\
\hline & A2 & From clustering of clusters to treatment station. \\
\hline & A3 & From total clustering of clusters to treatment station. \\
\hline \multirow{3}{*}{ Dilution } & B1 & $\begin{array}{l}\text { Individual and independent lines from cluster location to treatment } \\
\text { station. }\end{array}$ \\
\hline & B2 & From clustering of clusters to treatment station. \\
\hline & B3 & From total clustering of clusters to treatment station. \\
\hline \multirow{6}{*}{ Heat } & C1 & $\begin{array}{l}\text { Individual and independent lines from cluster location to treatment } \\
\text { station considering warming from wellhead. }\end{array}$ \\
\hline & $\mathbf{C 2}$ & $\begin{array}{l}\text { From clustering of clusters to treatment station considering warming } \\
\text { from wellhead. }\end{array}$ \\
\hline & $\mathbf{C 3}$ & $\begin{array}{l}\text { From total clustering of clusters to treatment station considering } \\
\text { warming from wellhead. }\end{array}$ \\
\hline & C4 & $\begin{array}{l}\text { Individual and independent lines from cluster location to treatment } \\
\text { station considering electric tracing. }\end{array}$ \\
\hline & C5 & $\begin{array}{l}\text { From clustering of clusters to treatment station considering electric } \\
\text { tracing. }\end{array}$ \\
\hline & C6 & $\begin{array}{l}\text { From total clustering of clusters to treatment station considering } \\
\text { electric tracing. }\end{array}$ \\
\hline
\end{tabular}

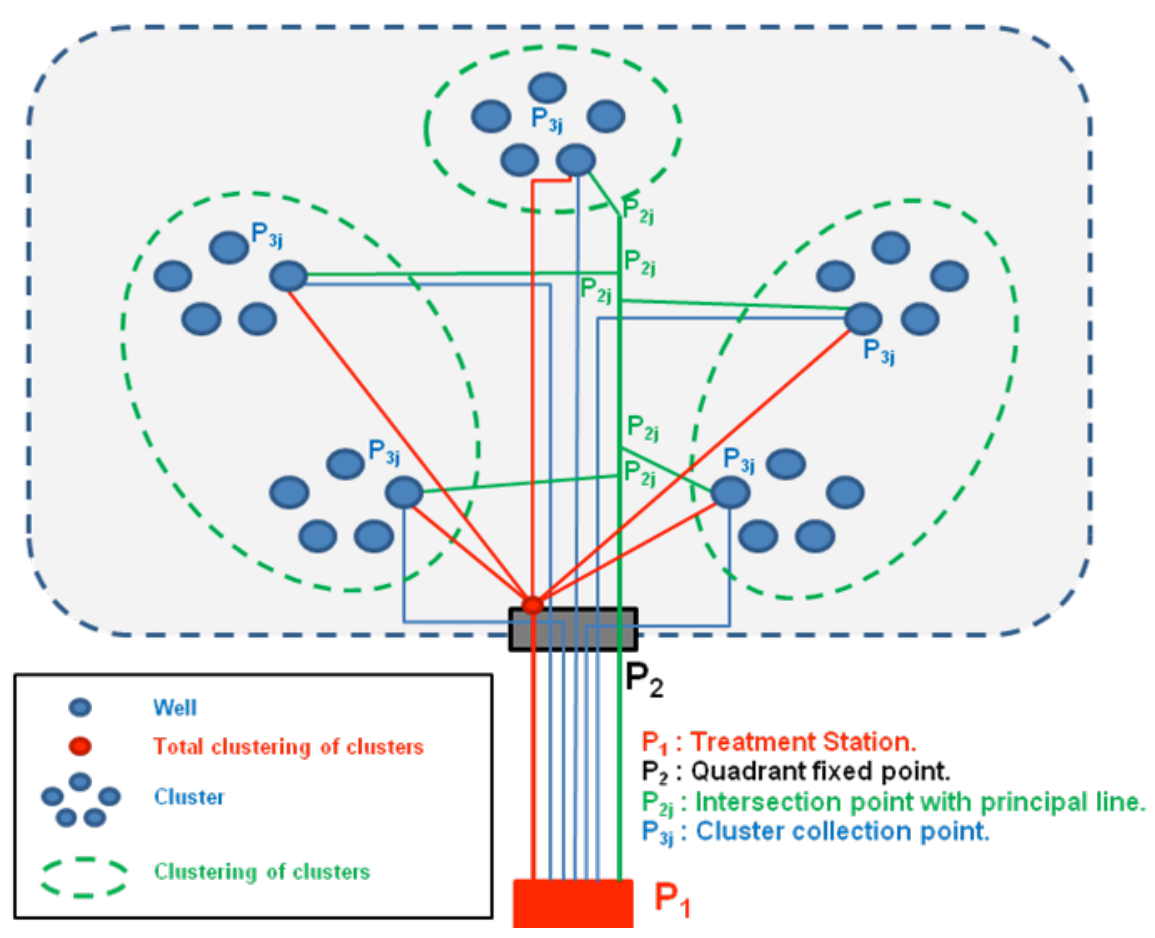


Figure 2. Unit of analysis - Quadrant structure

Once the unit of analysis was properly defined, the team began developing the models as follows.

\section{Model development and simulation design}

Figure 3 below summarizes the steps of the methodology designed for the analysis and selection of the different alternatives for oil gathering described above.

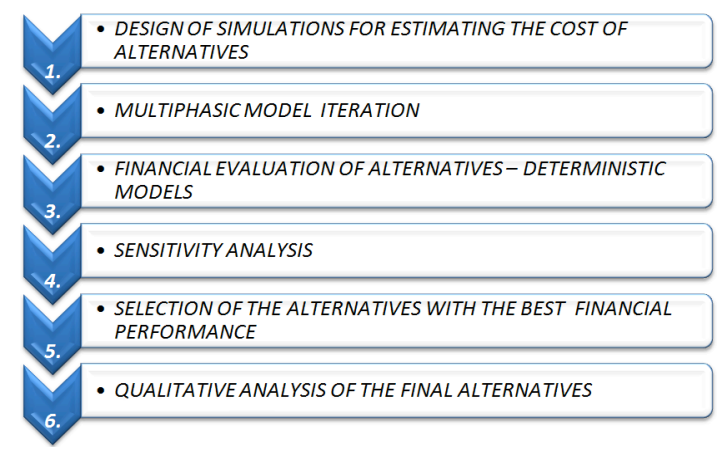

Figure 3. Methodology for the analysis and selection of alternatives

A detailed explanation of each step in this methodology is found below.

\subsection{Simulation design}

The design of a representative set of simulations was crucial, because the iteration process of the multiphase-model was too long and complex. In this way, after a depuration process, the unit of analysis (quadrant) was characterized by four main variables: Flow (BPD), Gas Oil ratio (GOR), Percentage of Water (WOR) and Temperature $\left({ }^{\circ} \mathrm{F}\right)$. ECOPETROL delivered the critical values for these variables under different main demand scenarios. These main scenarios are presented in Table 2.

Table 2. Characterization of main demand scenarios.

\begin{tabular}{|l|l|l|l|}
\hline & Minimum Demand & Medium Demand & Maximum Demand \\
\hline Flow (BPD) & 2000 & 10000 & 25000 \\
\hline GOR & 40 & 64,5 & 150 \\
\hline WOR (\%) & 40 & 67 & 96 \\
\hline Temperature ( $\left.{ }^{\circ} \mathbf{F}\right)$ & 180 & 160 & 120 \\
\hline
\end{tabular}

Additionally, in order to reduce the number of simulation scenarios to be considered (since there are 81 possible scenarios resulting from the main) the consulting group proposed to consider only three main scenarios. These scenarios were designed to cover all the range of possible outcomes, and they are described below (see Figure 4):

- Minimum Demand Scenario (MinD): Considers the four variables at the minimum demand level.

- Medium Demand Scenario (ED): Considers the four variables at the medium demand level.

- Maximum Demand Scenario (MaxE): Considers the four variables at the maximum demand level. 
The combination of the 12 alternatives, described in table 1, within each of the 3 main scenarios considered, produced 36 initial simulations. Each simulation was characterized by a fluid transport mechanism, an arrangement and the specific values for the quadrant's four main variables (Flow, GOR, WOR and Temperature). These simulations were made using the multiphase-model, which is described below, in order to obtain a detailed list of equipment and the supplies needed for performing the financial evaluation of alternatives.

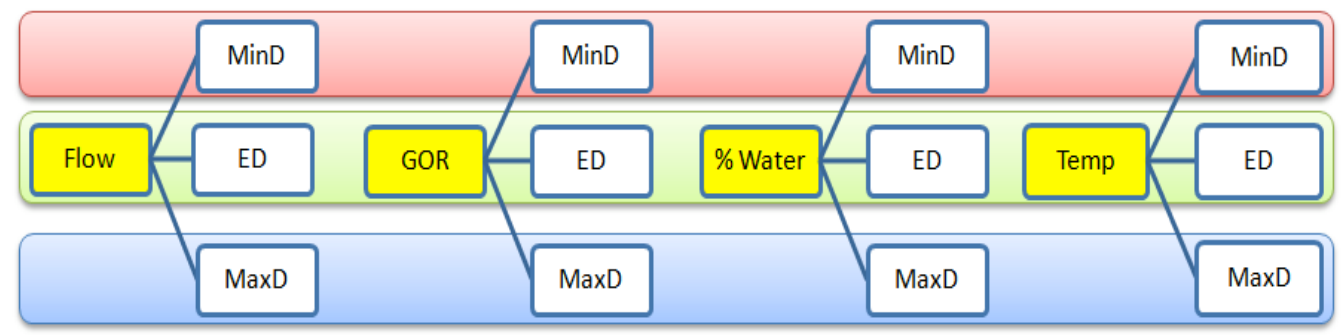

Figure 4. Simulation Design

\subsection{Multiphase model ${ }^{1}$}

After reviewing the relevant literature, the team decided to use an empirical thermodynamical model, which combines mass and energy balances: the Beggs and Brill model. The objective of the model is to find the energy requirements for fluid transportation from production wells to collection centers, taking into account fluid characteristics (temperature, density, viscosity), transportation volumes for each fluid considered and pipeline characteristics (line configurations, diameter, length and topography). Based on the model results, operational and capital costs can be estimated.

The original Beggs \& Brill (Beggs, 1973) correlation was used for pressure loss and the Beggs \& Brill Revised correlation was used to calculate hold-up.

Flow regime was determined with the Beggs \& Brill correlation. The correlation is based upon a flow regime map that is first determined supposing the flow is horizontal. A horizontal no slip hold-up is then calculated by correlations, and this hold-up is corrected for the angle of inclination.

The following enhancements to the original Beggs \& Brills method were made:

- An extra regime flow is considered which assumes a no-slip holdup,

- The friction factor is changed from the standard smooth pipe model, to a single-phase friction factor based on the average fluid speed.

For the pressure drop calculation, fluid properties such as density, viscosity and superficial tension were calculated for gas and liquid phase.

Considering the viscosity calculation is not an easy task in the case of heavy crude oil, the dead oil viscosity was based on lab data of Castilla and Chichimene oil. In addition, for the live oil viscosity, gas solubility was calculated using the Standing Method. The viscosity effects of water in the liquid phase were adjusted by the Woelfin correlation, which considers the oil/water inversion behavior as water cut

\footnotetext{
${ }^{1}$ This Model was developed by AB Proyectos, Bogotá, Colombia.
} 
function. Finally, for the temperature profile calculation a heat transfer global coefficient was applied to the buried pipe.

\subsection{Financial model}

Once the multiphase model was run for each of the 36 simulations, comparison criteria were determined. Although the financial factor is not the only relevant factor, it is a first approximation to the solution. In this sense, the financial model developed collects the results of the multiphase model (list of equipment and supplies) and, based on the implementation and operating periods of the fields, converts them into present monetary values. The structure of the financial model allows high flexibility in terms of the evaluation of alternatives, including all relevant monetary items with a moderate level of detail, considering the stage of project development ("before design"). In addition, the parameterized structure of the model allowed for performing sensitivity analysis on key financial items. Although this article does not show details of the financial sheets, their final objective can be clearly defined.

For each simulation, the total cost for each alternative scenario under consideration was found, in the following way:

$$
\begin{aligned}
& \text { Cost } A_{i}=f \text { (Pipes, Heating and Power stations, Power Requirement, Supplies, } \\
& \text { (Cost } A_{i} \mid \text { Minimum Demand) } \quad i=1, \ldots, 12 \\
& \text { (Cost } A_{i} \mid \text { Medium Demand) } \quad i=1, \ldots, 12 \\
& \text { (Cost } A_{i} \mid \text { Maximum Demand) } \quad i=1, \ldots, 12
\end{aligned}
$$

It is important to note that the comparison of the 12 alternatives was performed for each of the three scenarios as mentioned before.

\subsection{Qualitative model}

Sometimes quantitative methods by themselves are not sufficient for solving complex decision problems like the one presented in this article. Regarding this particular problem, given that multiple objectives must be taken into account, the use of a multi-objective model is extremely useful. The Analytic Hierarchy Process (AHP) (Saaty, 1994) is an adequate decision making model for this case. This model allows the decision maker to select the best alternative within a set of feasible alternatives, using a hierarchical structure representing the problem. In order to apply this type of model, the following steps must be taken:

1. Structure the decision problem as a hierarchy

2. Perform Pairwise comparisons among the elements of adjacent hierarchy levels

3. Combine the Pairwise comparisons to derive weights for hierarchical elements

4. Summarize the element weights into a set of ratings for decision alternatives.

The interaction between these three models (multiphase, financial, and qualitative) follows the sequence showed in Figure 5.

First the quadrant is fully characterized in order to adequately feed the multiphase model. Then, the output of the multiphase model is taken as the basis for the inputs of the Financial Analysis sheets. Finally, the final alternatives are compared using the qualitative model. 


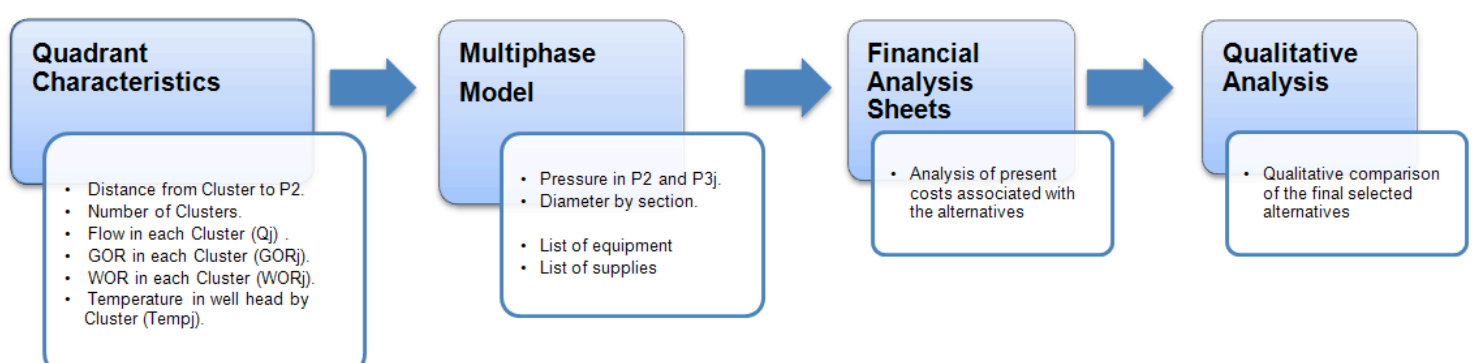

Figure 5. Information Flow

\section{Results and analysis}

All models were run based on estimates provided by ECOPETROL and the design of the simulations above. It must be pointed out that the quadrant used for the analysis was an eight-cluster quadrant with eight wells to each cluster. These clusters were equidistant $(8 \mathrm{~km})$ from the treatment station, due to arrangement modeling reasons. The other characteristics of the quadrant depended on the particular analysis scenario (see Table 2). The results for the financial model and the qualitative model are presented below.

\subsection{Financial evaluation}

The multiphase model in each simulation provided a list of equipment and supplies. This list was parameterized. Major aspects of investment as well as costs of operation and maintenance for the appropriate field's operation periods were considered. The present costs for each alternative were calculated through the cash flow obtained. This value is defined as the financial indicator for each alternative. Table 3 and figures 6,7 and 8 show the results for the twelve alternatives in each scenario.

Table 3. PV of the costs (US Dollars) for each alternative in each scenario.

\begin{tabular}{|l|l|l|l|l|l|}
\hline \multicolumn{3}{|c|}{ Alternatives Description } & \multicolumn{3}{c|}{ Demand Scenarios } \\
\hline Alt. & Mech. & Arrangement & Maximum & Medium & Minimum \\
\hline A1 & Power & $\begin{array}{l}\text { Individual and independent lines from cluster location to } \\
\text { treatment station. }\end{array}$ & $\$ 125,909,075$ & $\$ 32,456,557$ & $\$ 6,471,528$ \\
\hline A2 & Power & From clustering of clusters to treatment station. & $\$ 118,408,355$ & $\$ 10,985,369$ & $\$ 2,784,594$ \\
\hline A3 & Power & From total clustering of clusters to treatment station. & $\$ 127,340,394$ & $\$ 12,023,649$ & $\$ 3,386,173$ \\
\hline B1 & Dilution & $\begin{array}{l}\text { Individual and independent lines from cluster location to } \\
\text { treatment station. }\end{array}$ & $\$ 141,110,408$ & $\$ 41,730,797$ & $\$ 9,914,362$ \\
\hline B2 & Dilution & From clustering of clusters to treatment station. & $\$ 134,073,470$ & $\$ 19,198,524$ & $\$ 5,935,535$ \\
\hline B3 & Dilution & From total clustering of clusters to treatment station. & $\$ 142,706,005$ & $\$ 20,344,249$ & $\$ 6,921,542$ \\
\hline
\end{tabular}




\begin{tabular}{|l|l|l|l|l|l|}
\cline { 4 - 6 } C1 & Heat & $\begin{array}{l}\text { Individual and independent lines from cluster location to } \\
\text { treatment station considering warming from well-head. }\end{array}$ & $\$ 734,302,100$ & $\$ 79,847,319$ & $\$ 32,600,844$ \\
\hline C2 & Heat & $\begin{array}{l}\text { From clustering of clusters to treatment station considering } \\
\text { warming from well-head. }\end{array}$ & $\$ 731,453,294$ & $\$ 60,640,767$ & $\$ 28,933,387$ \\
\hline $\mathbf{C 3}$ & Heat & $\begin{array}{l}\text { From total clustering of clusters to treatment station considering } \\
\text { warming from well head. }\end{array}$ & $\$ 735,406,412$ & $\$ 59,200,935$ & $\$ 29,511,501$ \\
\hline C4 & Heat & $\begin{array}{l}\text { Individual and independent lines from cluster location to } \\
\text { treatment station considering electric tracing. }\end{array}$ & $\$ 376,220,507$ & $\$ 153,632,770$ & $\$ 67,760,796$ \\
\hline C5 & Heat & $\begin{array}{l}\text { From clustering of clusters to treatment station considering } \\
\text { electric tracing. }\end{array}$ & $\$ 359,484,647$ & $\$ 44,937,902$ & $\$ 23,710,689$ \\
\hline C6 & Heat & $\begin{array}{l}\text { From total clustering of clusters to treatment station considering } \\
\text { electric tracing. }\end{array}$ & $\$ 389,104,252$ & $\$ 56,655,103$ & $\$ 30,529,684$ \\
\hline
\end{tabular}

Analyzing the behavior of the twelve alternatives in each of the three scenarios considered (Minimum Demand, Medium Demand, and Maximum Demand), the Power mechanism and the Dilution mechanism dominate the Heating mechanisms (gas and tracing). This dominance is clear by the higher differences in the present value between these mechanisms. However, these differences are accentuated by increasing the level of demand in the scenario considered.

On the other hand, considering the pipe configuration, the arrangement 2 (from clustering of clusters to treatment station - Alternatives A2, B2, C2 and C5) shows a lower cost than the other arrangements. This difference is independent of the mechanism of transport considered. Furthermore, arrangement 1 (individual and independent lines from cluster location to treatment station) shows higher costs at the Maximum Demand and Medium Demand scenarios compared with the other two arrangements.

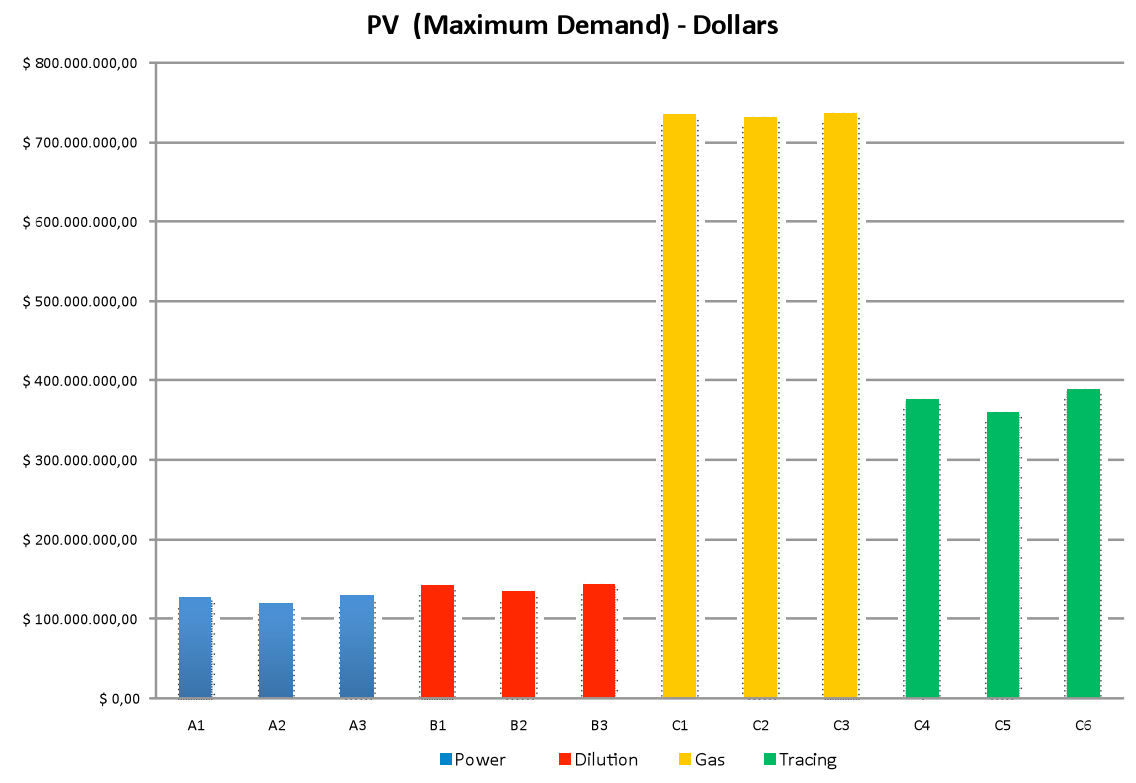

Figure 6. Present values at the maximum demand scenario 


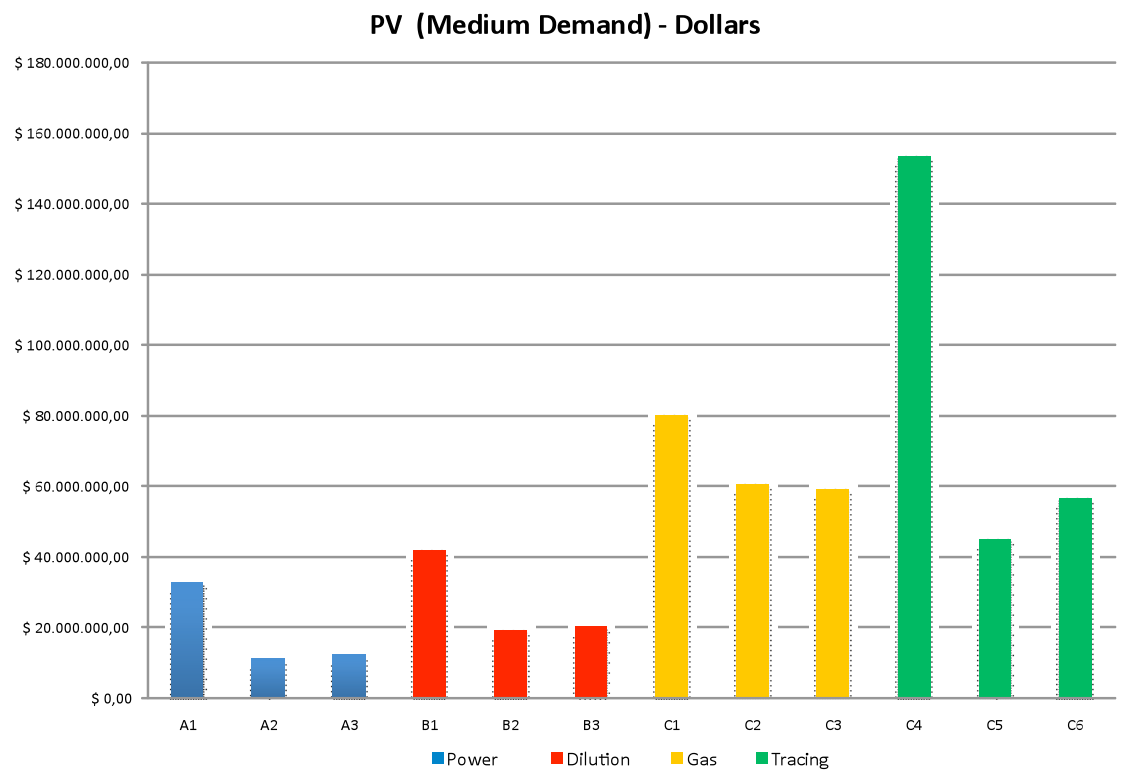

Figure 7. Present values at the medium demand scenario

\subsection{Sensitivity analysis}

Although the differences in the present costs of the alternatives were significant in the three scenarios considered, it is important to identify which of the financial aspects (investment, maintenance or operation) causes a significant impact on total costs. These aspects should then be characterized as random variables in order to perform a probabilistic analysis.

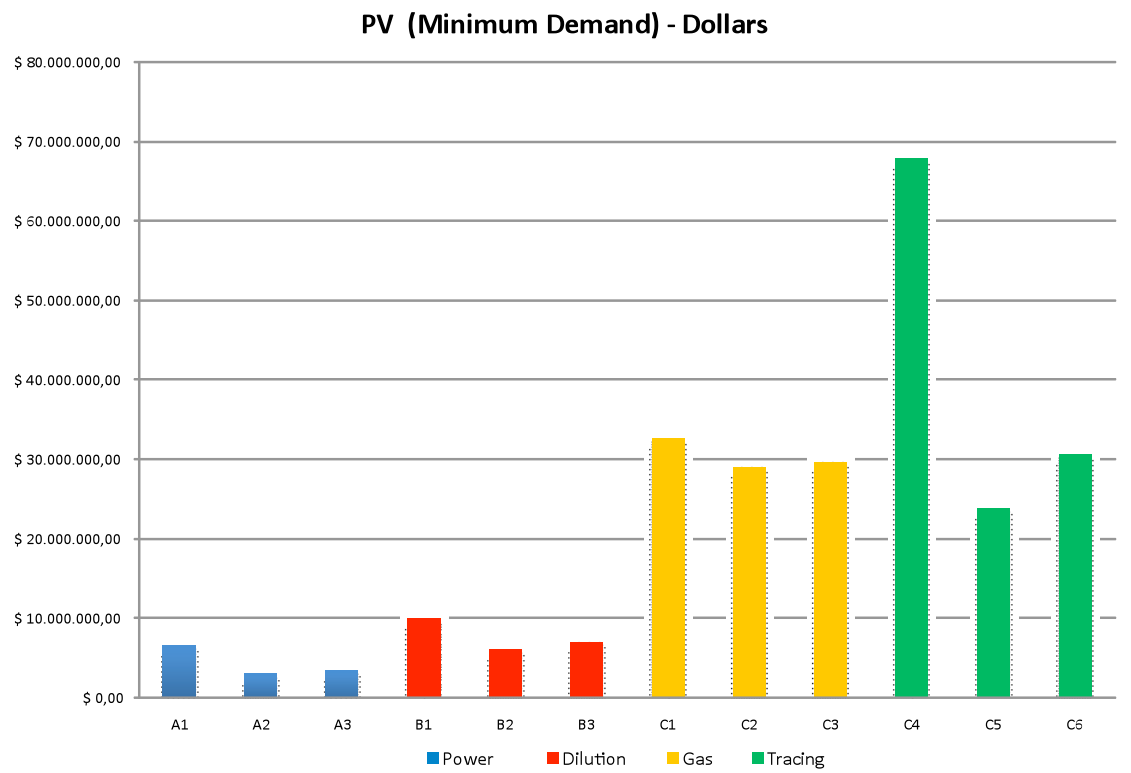

Figure 8. Present values at the minimum demand scenario

In order to identify those aspects, a significant variation (50\%) was applied to each aspect. However, the performance of the alternatives remained substantially the same. None of the financial aspects analyzed 
changes the ranking of the alternatives in any of the scenarios considered. For this reason, the probabilistic analysis was discarded.

The results of the financial models and sensitivity analysis were presented to ECOPETROL's decision group. This group, based on the results of the financial model and the latest available information from the fields, decided to consider as final alternatives only alternatives A2 and B2 (see Table 1). These two alternatives were then analyzed using the qualitative model.

\subsection{Qualitative analysis}

Figure 9 shows the final structure of the hierarchy for the qualitative evaluation of the two final alternatives under consideration. Some of the decision criteria described in chapter 3.3 were merged in order to reduce redundancy. Also, criteria that were evaluated as equal for the final alternatives were not taken into account.

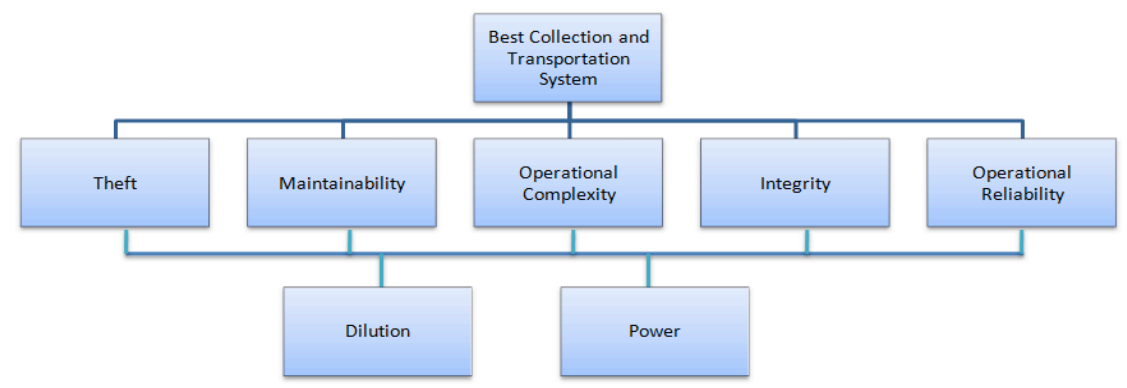

Figure 9. Hierarchical structure - Qualitative analysis

In order to evaluate the alternatives using the AHP, a workshop was organized with eleven engineers. They performed all the pairwise comparisons proposed. For the mathematical evaluation of the model, the team used Expert Choice 11.5. Figure 10 summarizes the behavior of each alternative regarding the goal (choose the best collection and transportation system) as well as the behavior of each criteria considered regarding the goal. According to the results obtained, the Dilution alternative was chosen, because it obtained a weight of almost twice the weight of the Power alternative.

A sensitivity analysis of the model, based on plausible changes in the comparisons, was performed. The analysis showed no significant changes in the final weights, so the model showed robustness in this sense.
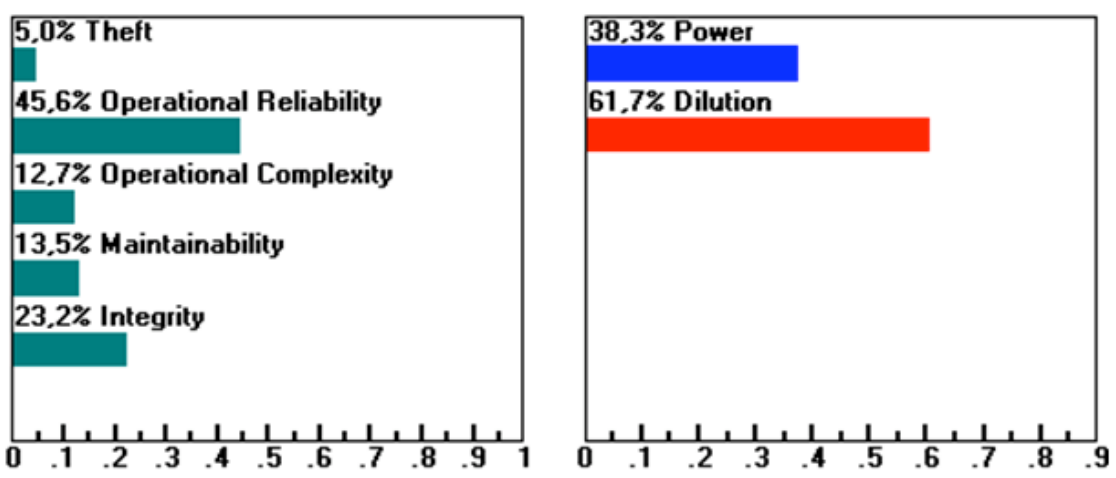

Figure 10. AHP Results 


\section{Discussion and conclusions}

\subsection{General conclusions}

1. A major contribution of this project was the general and specific methodologies used in structuring and analyzing the problem. Its success is mainly due to two aspects: the appropriate definition of the unit of analysis for the evaluation of alternatives, and the design of a representative set of simulations.

2. The unit of analysis represents in the best possible way the real situation of the fields with limited information by the time of project execution. This analysis unit has a very flexible configuration allowing several field representations to be tested.

3. The simulations were designed in such a way that they ensured coverage of the range of possible outcomes, reducing the great computational effort of using different models. The simulation design allowed the generalization of the plausible outcomes, which would not have been possible to explore through an exhaustive procedure.

4. The participation of a multidisciplinary team allowed for the interaction among different models. Therefore, the financial aspect was not the only one to be considered for the final evaluation of alternatives. However, this crucial aspect allowed for an initial depuration of the set of alternatives. This depuration reduced the number of final alternatives for the qualitative analysis.

\subsection{Regarding the results analysis}

5. Regarding the pipe arrangements, the clustering of clusters to treatment station arrangement showed a lower cost independently of the mechanism of transportation considered. This result remains unchanged under all the analysis scenarios as well as the sensitivity analysis.

6. In regard to the transportation mechanisms, Power and Dilution presented significant lower costs than Heating mechanisms. As with pipe arrangements, this happened for all analysis scenarios, showing robustness in the results.

7. It should be pointed out that the financial results presented in this article are not the total costs calculated for the fields. The costs shown served as a way of analyzing alternatives through a quadrant (unit of analysis). However, given the flexibility of the quadrant definition, the calculations may be easily extended in order to calculate total costs.

8. Once Heating mechanisms were excluded from a final analysis and the total clustering of clusters to treatment station arrangement was selected, the remaining alternatives, namely Power and Dilution, were compared on the basis of specific decision criteria. The Dilution mechanism showed a higher score than Power regarding almost all criteria. In the global qualitative comparison, Dilution obtained a weight twice higher than Power, which made it the preferred transportation mechanism for the decision making group.

9. Taking into account the financial and qualitative results, the decision making team selected the total clustering of clusters to treatment station arrangement and the dilution transportation mechanism as the best alternative for the problem analyzed.

\subsection{Regarding the impact of the project}

10. The design of a unit of analysis such as the one presented in this article, represents a high impact contribution since, due to its high flexibility, it becomes a scalable model that allows for an easy and adequate problem dimensioning, when initial information is scarce or insufficient. Thus, the major contribution of the analysis approach of this work lays in the fact that it makes possible to undertake the analysis at early stages of a problem involving decisions.

\section{REFERENCES}

Beggs H.D. \& Brill J.P. (1973). A study of two-phase flow in inclined pipes, J. Pet.Technol. 25, 607-617. 
Capen \& Vadcon. (2001). Probabilistic Reserves! Here at Last. SPE Reservoir Evaluation \& Engineering, 4(5), 387-394.

Castillo M. (2008). Toma de Decisiones en las Empresas: Entre el Arte y la Técnica. Metodologías, Modelos y Herramientas. Bogotá, Colombia: Ediciones UniAndes.

Newendorp \& Schuyler. (2000). Decision Analysis for petroleum Exploration, Aurora, CO: Planning Press.

Saaty \& Vargas. (1994) "Decisions Making in Economic, Political, Social and Technological Environments with the Analytic Hierarchy Process”. RSW Publications, Pittsburgh, PA. 